\title{
The Golden Ratio and its Effects on the Perceived Visual Appeal of Photos
}

\author{
Adem Gjonbalaj', Daniel Olivo ${ }^{1}$, Gwendolyn Lotter $^{1}$ and Matthew Dalzell ${ }^{1}$ \\ ${ }^{1}$ Bergen County Technical High School, Teterboro, NJ, USA
}

\section{$\underline{\text { ABSTRACT }}$}

The golden ratio (GR) has long been proposed to be the most visually appealing geometric proportion (Green, 1995). As a result, many popular sources have claimed that composition guidelines made from the GR such as the phi grid, the golden spiral, and the golden layout can be used to enhance the visual appeal of photographs. However, empirical evidence to substantiate this claim is inadequate. The purpose of this study is to analyze whether three GR composition guidelines, the phi grid, the golden spiral, and the golden layout, can create photo adaptations that are consistently perceived as more visually appealing than their non-golden ratio (NGR) counterparts. A digital survey was created and released on social media, targeting adults ages 18 through 64 with an interest in art. Respondents were presented with 9 pairs of photos, consisting of two photo adaptations, a GR version (made with one of the GR guidelines), and a NGR version. Respondents were instructed to choose the photo which they perceived to be more visually appealing. The results from the survey indicated that there was no inherent preference towards GR photos overall. However, when analyzing the results from each golden ratio guideline, it was found that only the golden layout produced GR photos that were more preferred than their NGR counterparts. Further research must be conducted to fully understand the aesthetic appeal of the golden layout. Until then, creative individuals should be skeptical of recommendations for using the GR to produce visual compositions.

\section{Literature Review}

\section{The Golden Ratio}

The GR is an irrational number, approximately 1.618, derived from two quantities when the longer segment (a) is to the smaller segment (b) as the smaller segment is to the sum of the larger and smaller segment $a / b=b / a+b(F i g u r e ~ 1)$. Alongside $\pi$ and e, the golden ratio is one of the most important irrational numbers in the field of mathematics as it is a common solution to many basic mathematical problems (Green, 1995). Regardless of the ratio's mathematical foundation, for centuries the ratio has been thought to contain intrinsic aesthetic appeal. Since the time of the Ancient Greeks and possibly long before, the GR was thought to be the most pleasing point in which to divide a line (Green, 1995). Since then, many have tried to substantiate the GR's alleged aesthetic appeal, by claiming the ratio to be apparent in many great pieces of artwork and nature. 


\section{The Golden Ratio}

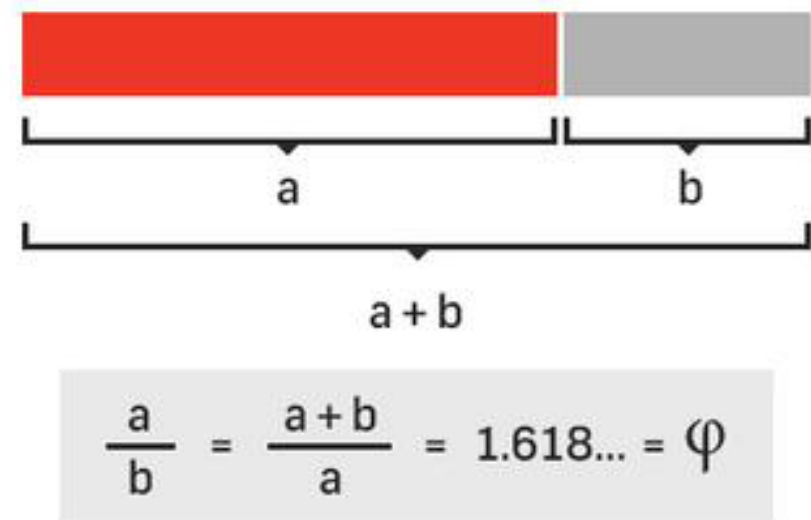

Figure 1. The Formulation of the Golden Ratio (2016) Retrieved from https://www.canva.com/learn/what-is-thegolden-ratio

\section{Aesthetic Appearances of the Golden Ratio}

For instance, a large proportion of books on the golden ratio have stated that the front dimensions of the Parthenon fit precisely into a golden rectangle, a rectangle whose side lengths are in the golden ratio (Livio, 2008). The GR has additionally been claimed to appear in the dimensions of the Great Pyramids of Giza, and in paintings such as the Mona Lisa and Ognissanti Madonna (Wittevenn, Witteveen, \& Childress, 2016; Livio, 2008). The aesthetic properties of the golden ratio have been further substantiated through claims regarding the ratio's ubiquity in nature. For example, the logarithmic spiral shape of the Chambered Nautilus has been said to grow by the proportion of $\Phi$, or the GR (Wells, 1992). The GR also allegedly appears in leaf and petal arrangements in flowers, aquatic life, bee's body segments, the solar system, and even in human anatomy (Akhtaruzzaman, 2012; Livio, 2008; Posamentier \& Lehmann, 2012). Literature that supports the GR's aesthetic appeal typically claims that the GR appears in both great works of art and nature.

\section{The Golden Ratio Debate}

These alleged appearances of the GR have, however, been subject to much debate. Subsequent inquiries have provided evidence to refute the aesthetic appearances of the GR. Of relevance here is the research of George Markowsky, a Professor of Computer Science and former employee at IBM's Thomas J. Watson Research Center who received his Ph.D in mathematics from Harvard University. Markowsky reasons that the purported appearances of the GR are supported by false/misconstrued evidence, and mathematical errors. For instance, Markowsky (1992) addresses the claim that the GR can be seen in the Parthenon stating, "The dimensions of the Parthenon vary from source to source probably because different authors are measuring between different points. With so many numbers available a golden ratio enthusiast could choose whatever number gave the best result" (p. 8). Markowsky additionally refutes the alleged appearances of the golden ratio in The Great Pyramid of Giza and in human anatomy, explaining that trying to measure complex structures gives individuals numerous lengths to choose from. With enough patience, these lengths can be misconstrued into the golden ratio (Markowsky, 1992). Many other researchers have similarly opposed the presence of the golden ratio such as Clement Falbo, a Mathematics Professor Emeritus at Sonoma State University. Falbo measured Nautilus Pompilius shells which are frequently stated to exhibit the golden ratio, and found that the average ratio of measurements was 1.33, and not the GR (1.618)(2005). Several other authors have contributed to the growing argument that the GR is in fact, not ubiquitous (Green, 2012; Steinbach, 1997; Fowler, 1982). This long-standing 
debate surrounding golden ratio appearances has prompted various authors throughout the years to test the aesthetic properties of the GR empirically.

\section{Empirical Studies on the Golden Ratio}

Known as one of the founders of experimental psychology, Gustav Fechner, a German philosopher, physicist and experimental psychologist, conducted the first empirical study on the GR. Fechner tested the aesthetic preference of the golden ratio in rectangles. 10 rectangles were placed in front of the participants and they were asked to choose the most pleasing, and least pleasing rectangle. The rectangles differed by their length-to-width ratios. $76 \%$ of the responses centered around preferring rectangles with the ratios 1.75, 1.62, and 1.50, with the most preferred rectangle having a length-to-width ratio equal to the GR (1.62). Hence, Fechner concluded that the golden rectangle was the most aesthetically pleasing rectangle (Fechner, 1971). In addition to Fechner's study, many subsequent studies have also found preferences for the GR using geometric stimuli (Benjafield, 2010; Godkewitsch, 1974). Simultaneously however, many studies have been published which have not found any preferences for the GR (Plug, 1980; Macrosson, 1997). Still, no consensus has been reached as to whether or not the golden ratio contains any aesthetic appeal.

\section{The Problem}

The crux of the matter however is that within the context of popular sources, the golden ratio is confidently being claimed as a useful tool which can enhance the visual appeal of photographs (Hegde, 2018; Staff, 2019). Claims regarding the aesthetic appeal of the GR in general have led several artists to already employ the GR in their work. French designer Malika Favre created an illustration of a woman walking by using a grid designed according to the GR. Other creatives such as Adrian Talbot, Jessica Nesbeth, Mark Hudson and even plastic surgeon Stephen R Marquardt have all used GR in their work (Bellos, 2014).

With no strong evidence concluding that the golden ratio contains aesthetic appeal, claims about the golden ratio's aesthetic properties might be misleading to creative individuals who plan on using this ratio. Yet still, popular sources frequently mention GR composition guidelines which creatives can use to allegedly create more visually appealing compositions. Commonly mentioned GR composition guidelines include the phi grid, the golden spiral and the golden layout. To inquire the truth as to whether these GR guidelines should be used by creatives, this research study aims to answer the question, "How effective is the phi grid, the golden spiral, and the golden layout at creating golden ratio photos that are perceived as more visually appealing than their non-golden ratio counterparts?"

Compared to the expansive number of studies which test the aesthetic appeal of the GR using geometric stimuli, there have been very few studies which test if the GR can enhance a photo's appearance. The greatest research effort on this issue is a study by Stefan Stieger and Viren Swami. Stefan Stieger is a professor in the Department of Psychology at Karl Landsteiner University of Health Sciences and Viren Swami is a professor of Social Psychology at Anglia Ruskin University. Their study examines whether the GR reflects an automatically elicited preference using the Implicit Association test. GR photos in their study were created by presenting the foreground object in the golden ratio. Preferences for the GR photos were compared to preferences for photos where the foreground object was presented in either the center, or in a $3 / 4$ ratio. The study found that there was no preference for the GR over other ratios (Stieger, 2015). Swami and Viren however only use one method to create GR photos. Therefore, it is illogical to claim that the GR cannot enhance photos when all other methods of its application have not been tested. To remedy this gap, this study will test if the GR can be used to create more visually appealing photos and will uniquely employ GR composition guidelines thought to create designs that are more pleasing to the eye. 


\section{Methodology}

\section{Summary of Method}

The purpose of this study was to test if photos which exhibited the GR were perceived as more visually appealing than NGR photos. A digital survey was created to quickly obtain responses from a large number of respondents, allowing for a more accurate analysis of the aesthetic effects the GR might have on images. The survey targeted adults ages 18 through 64 who had an interest in art. Within the survey, respondents were further randomly assigned to one of three sub-surveys. Each sub-survey contained 9 unique pairs of photos containing two photo adaptations, a GR version (made with one of the GR guidelines), and a NGR version. The respondents were then asked to choose the photo which they perceived to be more visually appealing. The results were analyzed with a chi square goodness of fit test, and a chi square test for homogeneity.

\section{Participants}

It was assumed that since the survey was art-related, respondents who were interested in art would have been more inclined to respond to the survey. To solidify this assumption, it was mentioned with the link to the survey that participants should be interested in art. It was also mentioned that only adults ages 18 through 64 should respond to the survey since children younger than 18 and the elderly older than 64 were considered as vulnerable populations by Bergen County Technical High School's Institutional Review Board. Respondents were trusted to fit within the requested demographics because their age or their interest in art could not be guaranteed.

\section{Stimuli}

For each of the three GR guidelines, (the phi grid, the golden spiral, and the GR layout), 9 pairs of photos were created, each pair consisting of a GR photo and a NGR photo. All photos were approved by Bergen County Technical High Schools Institutional Review Board to ensure that photos could not be interpreted as offensive or graphic. All the GR and NGR adaptations were made using Adobe Photoshop as well as the program PhiMatrix which was needed to produce the golden layouts. Each of the 9 pairs of photos were chosen and edited differently based on what GR guideline was used to create them.

\section{The Phi Grid}

The phi grid is a compositional overlay in the form of a $3 \times 3$ grid built from the GR. The phi grid contains the golden ratio because it splits up the image with three columns and rows in the ratio of 1:0.618:1 (for an example, see Figure 2). Photos can be edited with the phi grid by placing the focal point on either the lines or vertices of the phi grid, (for an example, see Figure 3). 


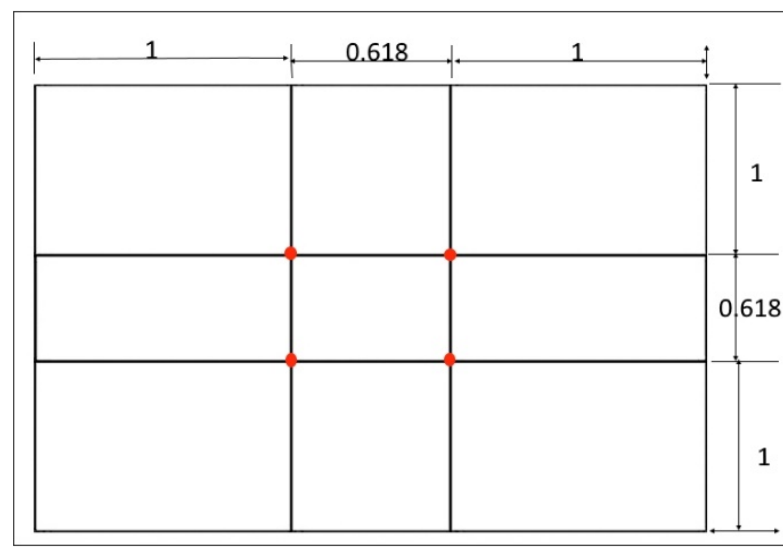

Figure 2. The Measurements of the Phi Grid (2017) Retrieved from https://ehabphotography.com/10-rules-for-photo-composition/

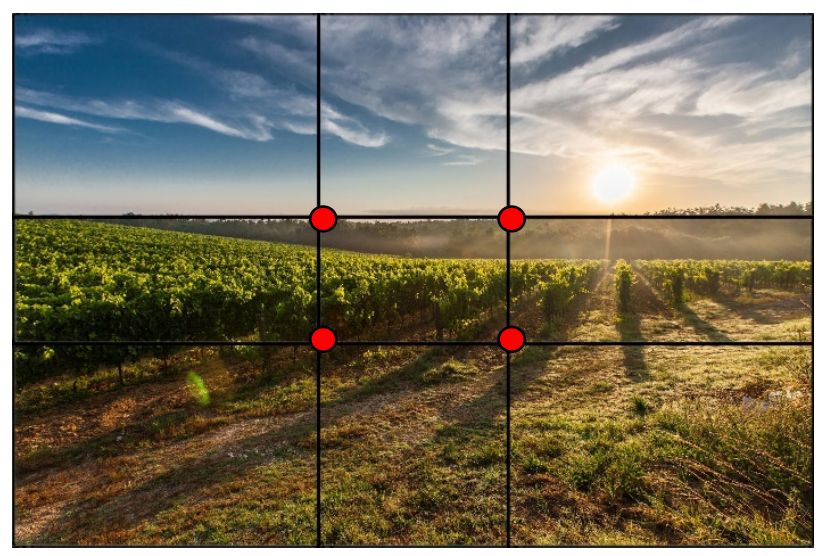

Figure 3. Photograph of a Field in Tuscany (2014). Retrieved from https://pixabay.com/photos/tuscanywinegrowing-grapevines-428041/

This photo's composition follows the phi grid since the horizon (one of the focal points), is placed on the uppermost horizontal line in the phi grid.

A common claim among many popular sources seems to be that the phi grid can be used to enhance the visual appeal of photos (Cousins, 2015; Elizarov, 2019; Brandon, 2019). To test the validity of these claims, 9 pairs of stimuli were created with the phi grid to be later judged by respondents. 9 photos were first selected from www.pixabay.com due to their wide variety of royalty free images. The 9 photos were selected based on how convenient the photos could be cropped so that the focal point/s would lay on the lines or vertices of the golden ratio. The focal points in the image were identified as the main object/s. For instance, in landscape photos, the focal point was usually chosen as the horizon, and if there was an additional vertical figure such as a human figure, it was also counted as a focal point. Only 1-2 focal points were chosen for images because more than 2 focal points made it difficult to crop all the focal points to the phi grid.

For the phi grid, if the long axis of the focal point was horizontal, then the photo was cropped so that the focal point would lay upon the closest horizontal line in the phi grid. If the long axis of the focal point was vertical, then the photo was cropped so that the focal point would lay upon the closest vertical line in the phi grid. If the focal point did not have a marked vertical or horizontal long axis, then the focal point was cropped so that the center laid on the closest vertice of the phi grid.

After the 9 GR photos were produced, copies of the photos were made to be turned into the NGR photos. This was done by shifting the photo content away from the border that was closest to the focal point. For example, if the horizon of a landscape was cropped to the lower horizontal line of the grid, then the horizon would be shifted up and away from the bottom border of the photo. It was made sure that the shifted focal point for the NGR photo never laid upon another line or vertice of the phi grid since this would create another GR photo. After 9 NGR adaptations were made from the 9 GR adaptations, a GR adaptation and a NGR adaptation of the same photo were placed in a pair, producing a total of 9 pairs of stimuli for the phi grid.

\section{The Golden Spiral}

Another GR composition guideline that has been claimed to create more visually appealing photos is the golden spiral. The golden spiral is a compositional overlay in the form of a logarithmic spiral made from the GR, (for an example, see Figure 4). Photos can be edited with the golden spiral by placing the center of the focal point on the innermost point of the spiral, (for an example, see figure 5). 


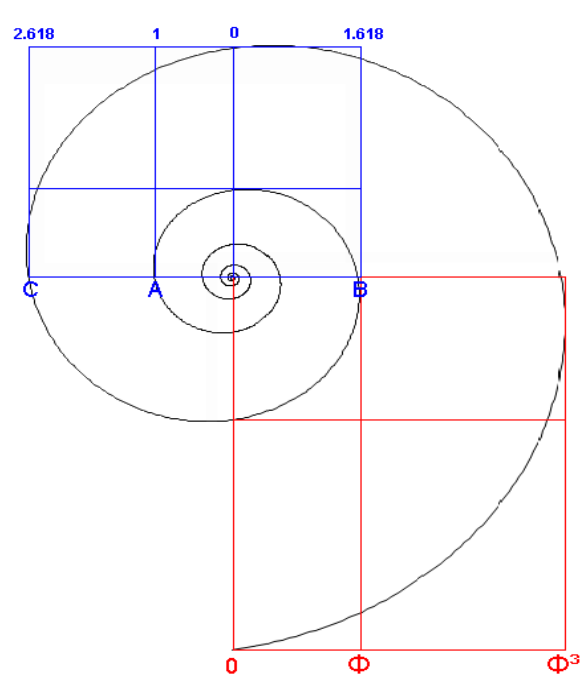

Figure 4. The Measurements of the Golden Spiral (2014). At point A, the spiral grows to a width of 1. Another 180 degree turn at point $B$ makes the spiral have a width of the GR (1.618).

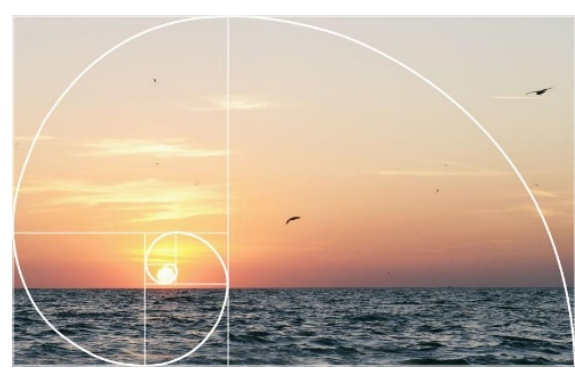

Figure 5. Photo of a Sunset that Follows the Golden Spiral (2017). This photo's composition follows the golden spiral since the sun (one of the focal points) is placed on the innermost point of the golden spiral.

Many popular sources have claimed that the innermost point of the golden spiral is where the eye naturally falls on and therefore composing/editing photos to the golden spiral can supposedly increase their visual appeal (Nurnberg, Leszun, Falconer, Olga, \& Gopee, 2018; Sparkman, 2016; Elizarov, 2019). To test if golden spiral photos are perceived as more visually appealing than NGR photos, 9 pairs of stimuli were created with the golden spiral to later be judged by respondents. 9 photos were first selected from www.pixabay.com based on how convenient the photos could be cropped so that the center of the focal point would lay upon the innermost point of the spiral. Unlike the photos used from the phi grid, only one focal point was chosen in the photos for the golden spiral. This was because the innermost point of the spiral was the only point where the focal point could be objectively cropped to.

After the 9 GR photos were created using the golden spiral, copies of the photos were made and turned into the NGR photos. Similar to how the NGR photos were produced for the phi grid, the content of the copied photos was shifted away from the closest border of the image. After 9 NGR adaptations were made from the 9 GR adaptations, a GR adaptation and a NGR adaptation of the same photo were placed in a pair, producing a total of 9 pairs of stimuli for the golden spiral.

\section{The Golden Layout}

The GR can also be used to create a two-column photo layout with the partition cropping the photos into the proportion of 1:0.681 (for an example, see Figure 6). 


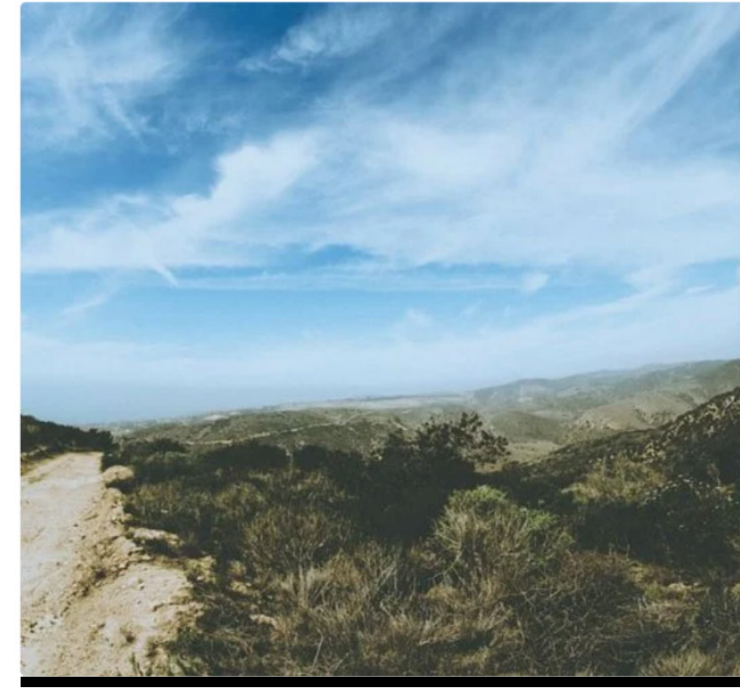

1

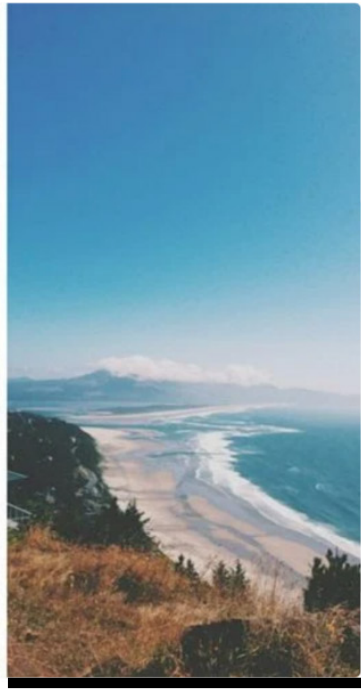

0.61

1.61

Figure 6. Two Column Layout of Landscape Photographs (2016). This photo is an example of a golden layout. The partition between the photos is creating the proportion of 1:0.618 with a sum of the golden ratio.

Since this usage of the GR has been recommended by popular sources but not yet given a name, this paper will address the produced layout as the golden layout for clarity (Gross, 2016; Soegaard, 2020). Since the golden layout contains two photos, 18 photos were chosen from www.pixabay.com. To create a golden layout, photos did not need to have a specific focal point. After the 18 photos were chosen, two photos were cropped so that when placed together, they would create a golden layout. Copies of the layouts were made and the NGR layouts were produced with three different techniques. $1 / 3$ of the NGR layout partitions were shifted directly in the center to create a symmetrical layout, another $\frac{1}{3}$ of the NGR layout partitions were shifted slightly to the right from the GR layout, and the last $1 / 3$ of the NGR layout partitions were shifted slightly to the left from the GR layout. This was done to produce NGR stimuli that were representative of the many ways layouts could be made not using the GR. After 9 NGR layouts were made from the 9 GR layouts, a GR layout and a NGR layout with the same photos were placed in a pair, producing a total of 9 pairs of layouts.

\section{Survey}

After all, 27 pairs of stimuli were produced, a digital survey was created. To retrieve responses quickly, the survey was posted on the social media platforms Reddit and Instagram.

The first section of the survey contained a consent form which explained the purpose of the survey, and asked individuals if they wished to consent to participating in the research study. The second section of the survey instructed the respondent to obtain a number from a link to a virtual 3-sided die. The respondent would then answer the question with the number they received from the link, and they would be taken to the corresponding sub-survey (survey 1 , survey 2, or survey 3). Each sub-survey was randomly assigned three pairs of stimuli from the phi grid, three pairs of stimuli from the golden spiral, and three pairs of golden layouts. These three sub-surveys were created since if all respondents took the same survey and a GR or NGR photo was more visually appealing due to elements other than 
the GR, then less respondents' choices would be affected by that one photo. The order that the stimuli pairs were presented in, as well as the placement of the GR photos in each pair, were randomized so that it would be extremely difficult for the respondents to notice which photos were made from the GR, and which were not. When taking the sub-survey, the respondents were asked to choose the photo which they perceived as more visually appealing for each of the 9 pairs. All participants who took the survey had no knowledge that the golden ratio was being tested due to the event that this would affect the participant's response.

\section{Data Analysis}

To test whether the GR photos were perceived as more visually appealing than the NGR photos, a chi square goodness of fit test was conducted between the overall GR and NGR preferences. To then determine which GR guidelines were effective at creating photos that were statistically perceived as more visually appealing, a chi square test for homogeneity was used. The chi square test for homogeneity tested if the GR guidelines differed in their preferences for the GR and NGR photos. After, three separate chi square goodness of fit tests were used to test the effectiveness of each GR guideline to create a statistically more preferred GR photo.

\section{Results}

The survey received 253 responses. Three respondents did not give consent to taking the survey therefore, those three responses were discarded. The respondents presumably fit into the demographics of adults ages 18 through 64 with an interest in art.

Table 1 and figure 7 depict the preferences in total for the GR and NGR photos. It is clear that the GR received the majority of preferences with 1158 overall preferences while the NGR received 1092 preferences. To test whether the GR photos were significantly more preferred than the NGR photos, a chi square test for goodness of fit was conducted. There was found to be no significant difference between the amount of GR preferences and the amount of NGR preferences, $\mathrm{X} 2(1, \mathrm{~N}=2250)=1.936, \mathrm{p}=.1641$. The golden ratio photos overall were therefore not consistently perceived as more visually appealing than the non-golden ratio photos.

Table 1. Overall Preferences for GR and NGR Photos

\begin{tabular}{|c|c|c|}
\hline & GR & NGR \\
\hline Preferences & 1158 & 1092 \\
\hline
\end{tabular}

GR and NGR Preferences

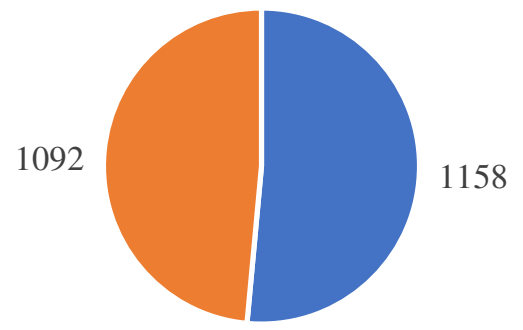

$\because \mathrm{GR}=\mathrm{NGR}$

Figure 7. Pie Chart for Overall GR and NGR Preferences 
Table 2 and figure 8 depict the preferences for the GR and NGR photos categorized by the GR composition guidelines. Although the overall GR photos were not statistically more preferred, there was still a possibility that at least one of the GR guidelines could have produced statistically more preferred GR photos. To figure this out, it first had to be tested if there was a difference in the proportions of GR and NGR preferences among the three GR guidelines.

To test if there was a difference in the proportions of GR and NGR votes among the three GR guidelines, a chi square test for homogeneity was conducted. The chi square test for homogeneity found that there was a significant difference between the distribution of GR and NGR votes, $\mathrm{X} 2(2, \mathrm{~N}=2250)=5.0, \mathrm{p}=.08$. It can be concluded that the GR categories did differ in their effectiveness in creating consistently more preferred GR photos.

Table 2. Preferences for GR and NGR Photos Categorized by Three Composition Guidelines

\begin{tabular}{|c|c|c|c|}
\hline & Phi Grid & Golden Spiral & Golden Layout \\
\hline GR Preferences & 380 & 368 & 410 \\
\hline NGR Preferences & 370 & 382 & 340 \\
\hline
\end{tabular}

Phi Grid

Preferences

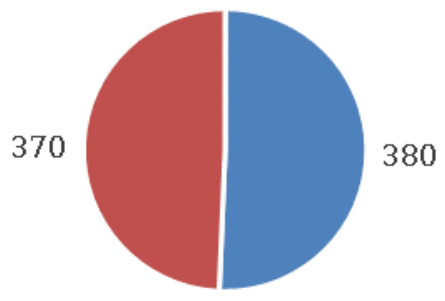

- GR $-\mathrm{NGR}$

\section{Golden Spiral}

Preferences

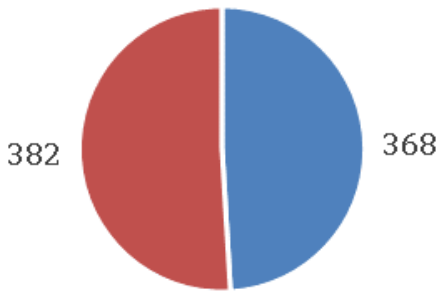

- GR - NGR
Golden Layout Preferences

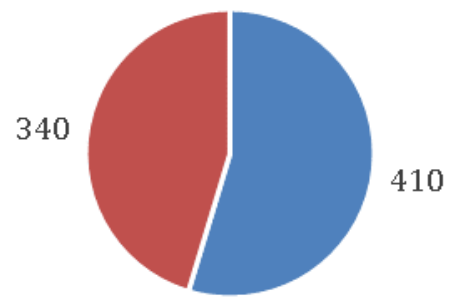

- GR - NGR

Figure 8. Pie Chart for Overall GR and NGR Preferences

To discover which golden ratio guideline produced GR photos that were statistically more preferred, three separate chi square goodness of fit tests were further conducted on each GR guideline.

For the phi grid, there was found to be no significant difference between the preferences for the GR and the NGR photos, $\mathrm{X}^{2}(1, \mathrm{~N}=750)=.13, \mathrm{p}=.715$. Therefore, the phi grid did not produce statistically more preferred GR photos.

For the golden spiral, there was also found to be no significant difference between the preference for the GR and NGR photos, $\mathrm{X}^{2}(1, \mathrm{~N}=750)=.261, \mathrm{p}=.609$. Therefore, the golden spiral did not produce statistically more preferred NGR photos.

For the golden layout, there was found to be a significant difference between the preference for the GR and NGR photos, $X^{2}(1, N=750)=6.533, p=.01059$. Therefore, the two-column golden layouts were statistically more preferred than the NGR layouts. Compared to the other golden ratio guidelines, the golden layout was the only effective guideline which created stimuli that were perceived as more visually appealing. 


\section{General Discussion}

The results from figures 5 and 6 indicate that the GR does not invariably create photos that are perceived as more visually appealing than other NGR photos. Although not all methods of the GR were tested in this study, it is evident through the phi grid and the golden spiral that the GR cannot be relied on to enhance the visual appeal of photographs until further studies are conducted. This conclusion is in line with a similar study by Stefan Stieger and Viren Swami which was aforementioned in the literature review. Their study tests if golden ratio photos are automatically more preferred when compared to photos which present the foreground object in ratios 1:2 and 3:4 (Stieger, 2015). The study finds no preference for photos which exhibit the GR. A possible explanation for the lack of preference for the GR photos in both Stieger's study and this study is that the difference between the GR photos and the NGR photos might have been too small to alter the appeal of the photos. This explanation has been voiced by George Markowsky (1992) who states, "I also experimented with a collection of 48 rectangles with ratios ranging from 1.6 to 1.7, which has convinced me that most people cannot see any differences among the rectangles whose ratios are so close together."(p. 18). Although this explanation refers to geometric stimuli, the differences between the GR and NGR might be especially more difficult to realize in photos since other factors such as visual balance and focal point placement affect the perceived visual appeal of photos. This possible explanation however should not replace the chance of the GR truly not having any aesthetic appeal.

Although no preference was seen for GR photos overall, the GR layouts were statistically perceived as more visually appealing than the NGR layouts. This indicates that rather than the GR being a universal law that governs aesthetic preference, the preference of the GR is extremely dependent on the composition guideline used. Past research using geometric stimuli has shown that the GR's appeal has been seen to be extremely sensitive to its methods of employment. Christopher Green published an extensive review of the research testing the aesthetic appeal of the GR. After reviewing many different results, Green came to the conclusion that the aesthetic effects of the golden ratio on geometric stimuli are extremely fragile and susceptible to different methods (Green 1995). Green's conclusion, although made from studies using geometric stimuli, seems to be the case for the GR in photography as well.

The current research on the GR and its effects on photos tend to indicate that there are no overarching preferences for the GR in photos. Although this may be true, this is not to say that some employable methods of the golden ratio cannot produce greatly more preferred photos, it just means that the GR's effects on the aesthetic appeal of photos does seem to be limited and dependent on the GR guideline being used.

\section{Implications}

The golden layouts were the only GR composition guidelines that were statistically perceived as more visually appealing than their NGR counterparts. However still, it cannot be said that the golden layout should be recommended to be used over all other layouts. The golden layouts only received 70 more preferences (410) than the NGR layouts (340) which means that around $45 \%$ of the respondents still preferred the NGR layouts more than the GR layouts. It can be seen that the golden layout is only mildly effective in creating photos that are perceived as statistically more visually appealing than their NGR counterparts. Therefore, the GR layout cannot be strongly recommended to be used over other layout methods until further research is done that finds a stronger preference for the GR layout. Considering the results for all three GR guidelines, creatives should be wary of recommendations which urge users to use the guidelines to enhance the visual appeal of photos.

\section{Limitations}

A limitation to this study is that the generalizability of the conclusion is uncertain. Studies have found that personality factors such as being an introvert or extrovert, as well as cultural identity can affect the preference for the golden ratio 
(Green 1995). Testing the demographic effects of GR preference was not the purpose of this study. The lack of demographic knowledge on the respondents in this study however reduces the generalizability of this conclusion, even more so for specific groups of individuals and especially for groups that are characterized by specific personalities or cultures.

A limitation that may have affected the responses is that a pair of a GR and NGR photo presented slightly different content of the same photo. This limitation was brought upon the study due to the method of production used to generate the photos, and the need to control for the size of the GR and NGR photos. The photos in this study were first cropped according to one of the GR guidelines. The sizes of the GR stimuli and the NGR stimuli had to be kept identical to reduce the inclusion of size as an independent variable. Therefore, the dimensions of the cropped GR photo were kept, and the focal point/partition was moved away from the GR. As the picture was moved away from the golden ratio, more elements of the photo could have been apparent in the NGR photo that were cropped out in the first GR photo. These other aesthetic elements could have skewed the photo's composition and could have affected the participants' decision on whether or not they preferred the GR photo.

Another limitation the method of cropping may have caused is that in some instances, a portion of the main object of the photo was cropped out. In one of the stimuli, a picture of a squirrel was cropped using the golden spiral where the squirrel's eyes were noted to be the focal point of the main object. When the content of the photo was then moved away from the GR, a part of the squirrel was cut off. The cropped part of the squirrel may have led a large portion of the respondents to choose the photo where more of the squirrely was being shown.

\section{Future directions}

Future studies should try to compose photos from other methods of the GR, different from the three that were used in this study (the phi grid, golden spiral, and golden layout). Because this study only tests three methods of the GR, the full extent of the GR's aesthetic preference in photos is unknown. Through testing other methods of the GR, a more holistic understanding can be reached on the aesthetic appeal of the GR in photography.

The aesthetic appeal of the GR through photography should continue to be tested however simultaneously, the aesthetic effects of the GR should also be tested through other mediums. Various complex stimuli such as web designs, paintings, product designs and other visual compositions can be used to test the GR. This will provide research with a better understanding of the full effects of the GR's aesthetic appeal not just in photos, but in many other aesthetic objects or designs.

\section{Acknowledgments}

Thank Mr. Olivo for your help with this project.

\section{References}

Akhtaruzzaman, M., \& Shafie, A. A. (2012). Geometrical Substantiation of Phi, the Golden Ratio and the Baroque of Nature, Architecture, Design and Engineering. International Journal of Arts, 1(1), 1-22. doi:

$10.5923 /$ j.arts. 20110101.01

Bellos, A. (2014, February 25). Golden Meaning: graphic artists depict the golden ratio - in pictures | Alex Bellos. Retrieved from https://www.theguardian.com/science/alexs-adventures-in-numberland/gallery/2014/feb/25/golden-meaning-graphic-artists-golden-ratio-pictures 
Benjafield, J. (1976). The Golden Rectangle: Some New Data. The American Journal of Psychology, 89(4), 737. doi: $10.2307 / 1421471$

Brandon, J. (2019, August 2). Divine Composition With Fibonacci's Ratio (The Rule of Thirds on Steroids). Retrieved from https://digital-photography-school.com/divine-composition-with-fibonaccis-ratio-the-rule-ofthirds-on-steroids/

Cousins, C. (2015, February 9). Looking at Images: Phi Grid vs. Rule of Thirds. Retrieved from https://designshack.net/articles/layouts/looking-at-images-phi-grid-vs-rule-of-thirds/

Elizarov, V. (2019, May 16). What is the Golden Ratio in Photography and How to Use It. Retrieved from https://www.phototraces.com/golden-ratio-in-photography/

Falbo, C. (2005). The Golden Ratio: A Contrary Viewpoint. The College Mathematics Journal, 36(2), 123. doi: $10.2307 / 30044835$

Fechner, G. (1971). Königliche Gesellschaft der Wissenschaften (Akademie der Wissenschaften) Göttingen, Bayerische Akademie der Wissenschaften München. doi: 10.1515/9783111433868

Fowler, D. H. (1982). A Generalization of the Golden Section. University of Warwick, Coventry, England . Retrieved from https://www.fq.math.ca/Scanned/20-2/fowler.pdf

Godkewitsch, M. (1974). The Golden Section: An Artifact of Stimulus Range and Measure of Preference. The American Journal of Psychology, 87(1/2), 269. doi: 10.2307/1422021

Green, C. D. (1995). All That Glitters: A Review of Psychological Research on the Aesthetics of the Golden Section. Perception, 24(8), 937-968. doi: 10.1068/p240937

Green, C. D. (2012). How to Find Refutations of the Golden Section without Really Trying. Empirical Studies of the Arts, 30(1), 115-122. doi: 10.2190/em.30.1.h

Gross, R. (2016, August 23). What is the golden ratio? What you need to know and how to use it. Retrieved from https://www.canva.com/learn/what-is-the-golden-ratio/

Livio, M. (2008). The Golden Ratio: the story of Phi, the world's most astonishing number. New York: Broadway Books.

Macrosson, W. D. K., \& Stewart, P. E. (1997). The Inclination of Artists to Partition Line Sections in the Golden Ratio. Perceptual and Motor Skills, 84(3), 707-713. doi: 10.2466/pms.1997.84.3.707

Markowsky, G. (1992). Misconceptions about the Golden Ratio. The College Mathematics Journal, 23(1), 2. doi: $10.2307 / 2686193$

Nurnberg, T., Leszun, J. J., Falconer, D., Olga, \& Gopee, T. (2018, March 6). How To Use the Golden Ratio To Improve Your Photography. Retrieved

Plug, C. (1980). The Golden Section Hypothesis. The American Journal of Psychology, 93(3), 467. doi: $10.2307 / 1422725$ 
Posamentier, A. S., \& Lehmann, I. (2012). The glorious golden ratio. Amherst, N.Y: Prometheus Books.

Soegaard, M. (2020, December). The Golden Ratio - Principles of form and layout. Retrieved from https://www.interaction-design.org/literature/article/the-golden-ratio-principles-of-form-and-layout

Sparkman, J. (2016, October 25). Why The Golden Ratio Is Better Than The Rule Of Thirds. Retrieved from https://petapixel.com/2016/10/24/golden-ratio-better-rule-thirds/

Staff, C. B. (2019, October 22). The Golden Ratio: A designer's guide. Retrieved from https://www.creativebloq.com/design/designers-guide-golden-ratio-12121546

Stieger, Stefan, and Viren Swami. "Time to Let Go? No Automatic Aesthetic Preference for the Golden Ratio in Art Pictures." Psychology of Aesthetics, Creativity, and the Arts, vol. 9, no. 1, 2015, pp. 91-100., doi:10.1037/a0038506.

Steinbach, P. (1997). Golden Fields: A Case for the Heptagon. Mathematics Magazine, 70(1), 22. doi: $10.2307 / 2691048$

Witteveen, W., Witteveen, A., \& Childress, D. H. (2016). The Great Pyramid of Giza: a modern view on ancient knowledge. Amsterdam: Frontier Publishing.

Wells, D. (1992). Hidden Connections Double Meanings. Cambridge: Cambridge University Press. 